\title{
Observer-Based Feedback Stabilization of Networked Control Systems with Random Packet Dropouts
}

\author{
Pengpeng Chen and Shouwan Gao \\ School of Computer Science and Technology, China University of Mining and Technology, Xuzhou 221116, China \\ Correspondence should be addressed to Shouwan Gao; gaoshouwan@gmail.com
}

Received 27 March 2013; Accepted 13 May 2013

Academic Editor: Tingwen Huang

Copyright (C) 2013 P. Chen and S. Gao. This is an open access article distributed under the Creative Commons Attribution License, which permits unrestricted use, distribution, and reproduction in any medium, provided the original work is properly cited.

\begin{abstract}
This paper is concerned with observer-based feedback stabilization of networked control systems (NCSs) with random packet dropouts. Both sensor-to-controller (S/C) and controller-to-actuator (C/A) packet dropouts are considered, and their behavior is assumed to obey the Bernoulli random binary distribution. The hold-input strategy is adopted, in which the previous packet is used if the packet is lost. An observer-based feedback controller is designed, and sufficient conditions for stochastic stability are derived in the form of linear matrix inequalities (LMIs). A numerical example illustrates the effectiveness of the results.
\end{abstract}

\section{Introduction}

In the past few years, dramatic progress has been made for network analysis. Unparticular, many important results have been obtained in engineering, such as the stability analysis and controller design for networked control systems (NCSs) [1-3] and consensus for networked multiagent systems [4, 5]. While NCSs have recently been receiving increasing attention due to their advantages over classical feedback control systems, the insertion of the communication network gives rise to new challenges. Among these challenges, time delays and packet losses are two important factors that can severely degrade the performance of NCSs. In the past few years, much attention has been paid to the time delay problem of NCSs, see [6-10], to name a few. Moreover, some existing control methods for time delays [11] can be used to NCSs with time delay. On the other hand, the control problem of NCSs with packet losses has also attracted considerable research interests (see, e.g., $[12,13]$ and references therein). This paper focuses on the impact of packet losses on the controller design for NCSs.

An arguably popular approach for modeling the packet loss phenomenon is to view the packet loss as a binary switching sequence according to a Bernoulli process which takes on values of zero and one with certain probability. Recently, some results have been obtained on such model
[15-17]. For example, in [15], the Kalman filtering problem is presented in the setting of intermittent observations and how the expected estimation error covariance depends on the tradeoff between loss probability and the system dynamics is showed. In [16], the problem of robust finite-horizon filtering is investigated for a class of uncertain systems with missing measurements. Moreover, in [17], the feedback stabilization schemes for discrete-time control systems with packet dropping network link are studied, and the feedback strategy presented is considerably simpler to implement.

It is noticed that, based on the Bernoulli distributed model, almost all the stability conditions and controller designs given in the aforementioned references are derived in terms of the assumption that the packet dropout exists only in the sensor-to-controller $(\mathrm{S} / \mathrm{C})$ side. The effect of controller-to-actuator (C/A) packet dropouts is neglected due to the complicated NCS modeling. Lately, there have appeared some research results which simultaneously consider S/C and C/A packet losses. In [14], the robust Ho control problem is considered for NCSs with both S/C and $\mathrm{C} / \mathrm{A}$ random communication packet losses. By modeling the random packet loss as a linear function of the stochastic variable satisfying Bernoulli binary distribution, stability analysis and controller synthesis problems are investigated. In [18], the observer-based Ho control problem is studied for discrete-time mixed delay systems with random packet 
dropouts and multiplicative noises. By modeling the packetloss phenomenon as Bernoulli distributed white sequences, the packet losses from S/C and from C/A are simultaneously considered. Furthermore, in [19], the similar problem is investigated for a class of networked nonlinear systems with global Lipschitz nonlinearities and random communication packet losses. In the above works, the zero-input strategy is adopted, in which, without considering the disturbance, the actuator (controller) input is set to zero when the C/A (S/C) packet is lost. However, for systems whose state and input signals change little from one time step to the next, for example, process control systems, the strategy may not perform well. In these situations, the hold-input strategy, that the latest packet stored in the buffer is used when the C/A or S/C packet is lost, gives a better performance [20]. To the best of the authors' knowledge, based on the hold-input strategy, the problem of observer-based feedback stabilization for NCSs with random $\mathrm{S} / \mathrm{C}$ and $\mathrm{C} / \mathrm{A}$ packet dropouts has not been investigated to date, which motivates the present study.

In this paper, the observer-based feedback stabilization problem for NCSs with both random S/C and C/A packet losses is considered. If the packet is lost, the hold-input strategy is adopted. Sufficient conditions for stochastic stability are given, and corresponding controller design steps are provided. An example is finally given to show the effectiveness of the control scheme proposed.

\section{Problem Formulation}

Consider the following NCS with random data packet dropouts shown in Figure 1, where sensors, controllers, and actuators are clock-driven:

$$
\begin{gathered}
x(k+1)=A x(k)+B u_{c}(k), \\
y_{c}(k)=\left(1-\alpha_{k}\right) C x(k)+\alpha_{k} y_{c}(k-1), \\
u_{c}(k)=\left(1-\beta_{k}\right) u(k)+\beta_{k} u_{c}(k-1),
\end{gathered}
$$

where $x(k) \in R^{n}$ is the state vector, $u_{c}(k) \in R^{m}$ is the control input to the actuator, $u(k) \in R^{m}$ is the desired control input computed by the controller, and $y_{c}(k) \in R^{p}$ is the measurement vector with transmission missing. $A, B$, and $C$ are known real constant matrices with appropriate dimensions. The stochastic variable $\alpha_{k}$ models the S/C packet loss: if the measurement packet is lost, then $\alpha_{k}=1$. Otherwise $\alpha_{k}=0$. Similarly, the stochastic variable $\beta_{k}$ models the $\mathrm{C} / \mathrm{A}$ packet loss: $\beta_{k}=1$ implies that the control packet is lost, and $\beta_{k}=0$ implies that the control packet is correctly delivered. $\left(\alpha_{k}, \beta_{k}\right)$ are independent identically distributed (i.i.d.) Bernoulli random variables with

$$
\begin{aligned}
& \operatorname{prob}\left\{\alpha_{k}=1\right\}=E\left\{\alpha_{k}\right\}=\bar{\alpha}, \\
& \operatorname{prob}\left\{\beta_{k}=1\right\}=E\left\{\beta_{k}\right\}=\bar{\beta} .
\end{aligned}
$$

The buffers store only one packet, and they will be updated if a new packet arrives. By such a mechanism, the buffers always store the most recent packet and are used for the purpose of packet dropout compensation. For buffer 1, if the measurement packet is correctly delivered, then $\alpha_{k}=0$, that is, $y_{c}(k)=C x(k)$, while if the packet is lost, $\alpha_{k}=1$, that is, $y_{c}(k)=y_{c}(k-1)$. This observation model is summarized by (2). For buffer 2 , if the packet is correctly delivered, then $\beta_{k}=$ 0 , that is, $u_{c}(k)=u(k)$. Otherwise, the actuator will employ the previous control value, that is, $\beta_{k}=1, u_{c}(k)=u_{c}(k-1)$, as suggested in [21]. This compensation scheme is summarized by (3).

The dynamic observer-based control scheme for the system is described as follows:

$$
\begin{gathered}
\widehat{x}(k+1)=A \hat{x}(k)+B u_{c}(k) \\
+L\left[y_{c}(k)-(1-\bar{\alpha}) C \hat{x}(k)-\bar{\alpha} y_{c}(k-1)\right], \\
u(k)=K \hat{x}(k),
\end{gathered}
$$

where $\widehat{x}(k) \in R^{n}$ is the state estimation and $L \in R^{n \times p}$ and $K \in R^{m \times n}$ are the observer and controller gains, respectively.

Let the estimation error be

$$
e(k)=x(k)-\widehat{x}(k),
$$

and by substituting (2), (3), and (5) to (1) and (6), we have

$$
\begin{aligned}
x(k+1)= & {[A+(1-\bar{\beta}) B K] x(k) } \\
& -(1-\bar{\beta}) B K e(k)+\bar{\beta} B u_{c}(k-1) \\
& -\left(\beta_{k}-\bar{\beta}\right) B K x(k)+\left(\beta_{k}-\bar{\beta}\right) B K e(k) \\
& +\left(\beta_{k}-\bar{\beta}\right) B u_{c}(k-1), \\
e(k+1)= & {[A-(1-\bar{\alpha}) L C] e(k) } \\
& +\left(\alpha_{k}-\bar{\alpha}\right) L C x(k)-\left(\alpha_{k}-\bar{\alpha}\right) L y_{c}(k-1) .
\end{aligned}
$$

Moreover, we can rewrite (2) and (3) as

$$
\begin{aligned}
y_{c}(k)= & (1-\bar{\alpha}) C x(k)+\bar{\alpha} y_{c}(k-1) \\
& -\left(\alpha_{k}-\bar{\alpha}\right) C x(k)+\left(\alpha_{k}-\bar{\alpha}\right) y_{c}(k-1), \\
u_{c}(k)= & (1-\bar{\beta}) K x(k)-(1-\bar{\beta}) K e(k)+\bar{\beta} u_{c}(k-1) \\
& -\left(\beta_{k}-\bar{\beta}\right) K x(k)+\left(\beta_{k}-\bar{\beta}\right) K e(k) \\
& +\left(\beta_{k}-\bar{\beta}\right) u_{c}(k-1) .
\end{aligned}
$$

By defining

$$
z(k)=\left[\begin{array}{llll}
x^{T}(k) & e^{T}(k) & y_{c}^{T}(k-1) & u_{c}^{T}(k-1)
\end{array}\right]^{T},
$$

(7)-(8) can be rewritten in a compact form as follows:

$$
z(k+1)=\left(\Phi_{1}+\Phi_{2}\right) z(k)
$$


where

$$
\begin{gathered}
\Phi_{1}=\left[\begin{array}{cccc}
A+(1-\bar{\beta}) B K & -(1-\bar{\beta}) B K & 0 & \bar{\beta} B \\
0 & A-(1-\bar{\alpha}) L C & 0 & 0 \\
(1-\bar{\alpha}) C & 0 & \bar{\alpha} & 0 \\
(1-\bar{\beta}) K & -(1-\bar{\beta}) K & 0 & \bar{\beta}
\end{array}\right], \\
\Phi_{2}=\left[\begin{array}{cccc}
-\left(\beta_{k}-\bar{\beta}\right) B K & \left(\beta_{k}-\bar{\beta}\right) B K & 0 & \left(\beta_{k}-\bar{\beta}\right) B \\
\left(\alpha_{k}-\bar{\alpha}\right) L C & 0 & -\left(\alpha_{k}-\bar{\alpha}\right) L & 0 \\
-\left(\alpha_{k}-\bar{\alpha}\right) C & 0 & \alpha_{k}-\bar{\alpha} & 0 \\
-\left(\beta_{k}-\bar{\beta}\right) K & \left(\beta_{k}-\bar{\beta}\right) K & 0 & \beta_{k}-\bar{\beta}
\end{array}\right] .
\end{gathered}
$$

Since $\alpha_{k}$ and $\beta_{k}$ are stochastic variables, we need to introduce the following definition before proceeding further.

Definition 1. System (1) is said to be mean square stable if for any $\varepsilon>0$, there is a $\delta(\varepsilon)>0$ such that $E\left\{|x(k)|^{2}\right\}<\varepsilon, k>0$ when $E\left\{|x(0)|^{2}\right\}<\delta(\varepsilon)$. In addition, if $\lim _{k \rightarrow \infty} E\left\{|x(k)|^{2}\right\}=0$ for any initial conditions, the system (1) is said to be globally mean-square asymptotically stable (GMSAS).

\section{Main Results}

In this section, we shall discuss the observer-based feedback controller design problem for (1). Without loss of generality, we make the following assumption.

Assumption 2. The matrix $B \in R^{n \times m}$ is of full-column rank; that is, $\operatorname{Rank}(B)=m$.

For the matrix $B \in R^{n \times m}$ being of full-column rank, there always exist two orthogonal matrices $U \in R^{n \times n}$ and $V \in R^{m \times m}$, such that

$$
U B V=\left[\begin{array}{l}
U_{1} \\
U_{2}
\end{array}\right] B V=\left[\begin{array}{c}
\Lambda \\
0
\end{array}\right]
$$

where $U_{1} \in R^{m \times n}, U_{2} \in R^{(n-m) \times n}$ and $\Lambda=$ $\operatorname{diag}\left\{\begin{array}{llll}\lambda_{1} & \lambda_{2} & \cdots & \lambda_{m}\end{array}\right\}$, in which $\lambda_{i}(i=1,2, \ldots, m)$ are nonzero singular values of $B$.

Furthermore, in order to derive the main result, the following lemma will be needed.

Lemma 3 (see [22] ). Let the matrix $B \in R^{n \times m}$ be of fullcolumn rank. If matrix $P \in R^{n \times n}$ is of the following structure

$$
P=U^{T}\left[\begin{array}{cc}
P_{1} & 0 \\
0 & P_{2}
\end{array}\right] U=U_{1}^{T} P_{1} U_{1}+U_{2}^{T} P_{2} U_{2}
$$

where $P_{1} \in R^{m \times m}>0, P_{2} \in R^{(n-m) \times(n-m)}>0$, and $U_{1}, U_{2}$ are defined in (12); then there exists a nonsingular matrix $\widetilde{P} \in$ $R^{m \times m}$ such that $B \widetilde{P}=P B$.

Theorem 4. Consider the networked control problem in Figure 1. Given the dynamic observer-based control scheme (5), system (1) is GMSAS for any S/C and C/A random data packet dropouts if there exist matrices $P>0, S>0, M>0, N>$ $0, L$, and $K$ satisfying

$$
\left[\begin{array}{ccc}
\Omega_{1} & \Pi_{1} & \Pi_{2} \\
* & \Omega_{1}^{-1} & 0 \\
* & * & \Omega_{2}^{-1}
\end{array}\right]<0,
$$

where

$$
\begin{aligned}
& \Omega_{1}=\operatorname{diag}\{-P-S-M-N\} \text {, } \\
& \Pi_{1}=\Phi_{1}^{T}, \\
& \Pi_{2}=\left[\begin{array}{cccc}
B K & -B K & 0 & -B \\
L C & 0 & -L & 0 \\
C & 0 & -I & 0 \\
K & -K & 0 & -I
\end{array}\right]^{T}, \\
& \Omega_{2}=\operatorname{diag}\left\{\begin{array}{llll}
-\alpha_{1}^{2} P & -\alpha_{2}^{2} S & -\alpha_{2}^{2} M & -\alpha_{1}^{2} N
\end{array}\right\} \text {, }
\end{aligned}
$$

in which $\alpha_{1}=[\bar{\beta}(1-\bar{\beta})]^{1 / 2}, \alpha_{2}=[\bar{\alpha}(1-\bar{\alpha})]^{1 / 2}$.

Proof. To prove the theorem, we introduce the following functions:

$$
\begin{gathered}
V(z(k), k)=V_{1}+V_{2}+V_{3}+V_{4}, \\
V_{1}=x^{T}(k) P x(k), \\
V_{2}=e^{T}(k) \operatorname{Se}(k), \\
V_{3}=y_{c}^{T}(k-1) M y_{c}(k-1), \\
V_{4}=u_{c}^{T}(k-1) N u_{c}(k-1),
\end{gathered}
$$

where $P, S, M$, and $N$ are positive definite matrices to be determined. Then, we have

$$
\begin{aligned}
E\{\Delta V \mid & z(k), \ldots, z(0)\} \\
= & E\{V(z(k+1), k+1) \mid z(k), \ldots, z(0)\} \\
& -V(z(k), k) \\
= & E\left\{\Delta V_{1} \mid z(k), \ldots, z(0)\right\} \\
& +E\left\{\Delta V_{2} \mid z(k), \ldots, z(0)\right\} \\
& +E\left\{\Delta V_{3} \mid z(k), \ldots, z(0)\right\} \\
& +E\left\{\Delta V_{4} \mid z(k), \ldots, z(0)\right\},
\end{aligned}
$$


where

$$
\begin{aligned}
& E\left\{\Delta V_{1} \mid z(k), \ldots, z(0)\right\} \\
& =\{[A+(1-\bar{\beta}) B K] x(k)-(1-\bar{\beta}) B K e(k) \\
& \left.+\bar{\beta} B u_{c}(k-1)\right\}^{T} P \\
& \times\{[A+(1-\bar{\beta}) B K] x(k)-(1-\bar{\beta}) B K e(k) \\
& \left.+\bar{\beta} B u_{c}(k-1)\right\} \\
& +E\left\{\left(\beta_{k}-\bar{\beta}\right)^{2}\right\}\left[B K x(k)-B K e(k)-B u_{c}(k-1)\right]^{T} P \\
& \times\left[B K x(k)-B K e(k)-B u_{c}(k-1)\right]-x^{T}(k) P x(k), \\
& E\left\{\Delta V_{2} \mid z(k), \ldots, z(0)\right\} \\
& =\{[A-(1-\bar{\alpha}) L C] e(k)\}^{T} S\{[A-(1-\bar{\alpha}) L C] e(k)\} \\
& +E\left\{\left(\alpha_{k}-\bar{\alpha}\right)^{2}\right\}\left[L C x(k)-L y_{c}(k-1)\right]^{T} \\
& \times S\left[L C x(k)-L y_{c}(k-1)\right]-e^{T}(k) \operatorname{Se}(k), \\
& E\left\{\Delta V_{3} \mid z(k), \ldots, z(0)\right\} \\
& =\left\{(1-\bar{\alpha}) C x(k)+\bar{\alpha} y_{c}(k-1)\right\}^{T} \\
& \times M\left\{(1-\bar{\alpha}) C x(k)+\bar{\alpha} y_{c}(k-1)\right\} \\
& +E\left\{\left(\alpha_{k}-\bar{\alpha}\right)^{2}\right\}\left[C x(k)-y_{c}(k-1)\right]^{T} \\
& \times M\left[C x(k)-y_{c}(k-1)\right] \\
& -y_{c}^{T}(k-1) M y_{c}(k-1), \\
& E\left\{\Delta V_{4} \mid z(k), \ldots, z(0)\right\} \\
& =\left\{(1-\bar{\beta}) K x(k)-(1-\bar{\beta}) K e(k)+\bar{\beta} u_{c}(k-1)\right\}^{T} N \\
& \times\left\{(1-\bar{\beta}) K x(k)-(1-\bar{\beta}) K e(k)+\bar{\beta} u_{c}(k-1)\right\} \\
& +E\left\{\left(\beta_{k}-\bar{\beta}\right)^{2}\right\}\left[K x(k)-K e(k)-u_{c}(k-1)\right]^{T} N \\
& \times\left[K x(k)-K e(k)-u_{c}(k-1)\right] \\
& -u_{c}^{T}(k-1) N u_{c}(k-1) \text {. }
\end{aligned}
$$

Noting that $E\left\{\left(\alpha_{k}-\bar{\alpha}\right)^{2}\right\}=\bar{\alpha}(1-\bar{\alpha})$ and $E\left\{\left(\beta_{k}-\bar{\beta}\right)^{2}\right\}=$ $\bar{\beta}(1-\bar{\beta})$, we obtain

$$
E\{\Delta V \mid z(k), \ldots, z(0)\}=z^{T}(K) \Sigma z(k),
$$

in which

$$
\Sigma=\Omega_{1}-\Pi_{1} \Omega_{1} \Pi_{1}^{T}-\Pi_{2} \Omega_{2} \Pi_{2}^{T} .
$$

By the Schur complement, (14) guarantees $\Sigma<0$. Thus, for all $z(k) \neq 0$, it is easy to know that

$$
E\{V(z(k+1), k+1) \mid z(k), \ldots, z(0)\}-V(z(k), k)<0 .
$$

That is, there exists $0<\gamma<1$ satisfying

$$
E\{V(z(k+1), k+1) \mid z(k), \ldots, z(0)\} \leq \gamma V(z(k), k) .
$$

Using the smoothing property

$$
\begin{aligned}
& E\{V(z(k), k) \mid z(k-2), \ldots, z(0)\} \\
& =E\{E\{V(z(k), k) \mid z(k-1), \ldots, z(0)\} \mid \\
& \quad z(k-2), \ldots, z(0)\}
\end{aligned}
$$

in (22) after taking the conditional expectation, we can conclude

$$
\begin{aligned}
E\{V & (z(k), k) \mid z(k-2), \ldots, z(0)\} \\
& \leq \gamma E\{V(z(k-1), k-1) \mid z(k-2), \ldots, z(0)\} \\
& \leq \gamma^{2} V(z(k-2), k-2) .
\end{aligned}
$$

Continuing this process, we get

$$
E\{V(z(k), k)\} \leq \gamma^{k} V(z(0), 0),
$$

which implies that

$$
\begin{aligned}
E\left\{\sum_{k=0}^{N} V(z(k), k)\right\} & \leq\left(1+\gamma+\cdots+\gamma^{N}\right) V(z(0), 0) \\
& =\frac{1-\gamma^{N+1}}{1-\gamma} V(z(0), 0) .
\end{aligned}
$$

Since $S, M$, and $N$ are positive definite matrices, it is easy to conclude that

$$
\lim _{N \rightarrow \infty} E\left\{\sum_{k=0}^{N} x^{T}(k) x(k)\right\} \leq \frac{1}{(1-\gamma) \lambda_{\min }(P)} V(z(0), 0),
$$

which implies $\lim _{N \rightarrow \infty} E\left\{|x(k)|^{2}\right\}=0$, and the proof is completed.

Note that the condition (14) is not an LMI, hence, cannot be solved by MATLAB LMI Toolbox. In the following, we will deal with the controller design problem and derive the explicit expression of the controller parameters in terms of LMI.

Theorem 5. Consider the networked control problem in Figure 1. The dynamic observer-based control scheme (5) exists such that system (1) is GMSAS for any $S / C$ and $C / A$ random data packet dropouts if there exist matrices $P_{1}>0, P_{2}>0$, $S>0, M>0, G$, and $H$, satisfying

$$
\left[\begin{array}{ccc}
\Omega_{1} & \Xi_{1} & \Xi_{2} \\
* & \Omega_{1} & 0 \\
* & * & \Omega_{1}
\end{array}\right]<0,
$$




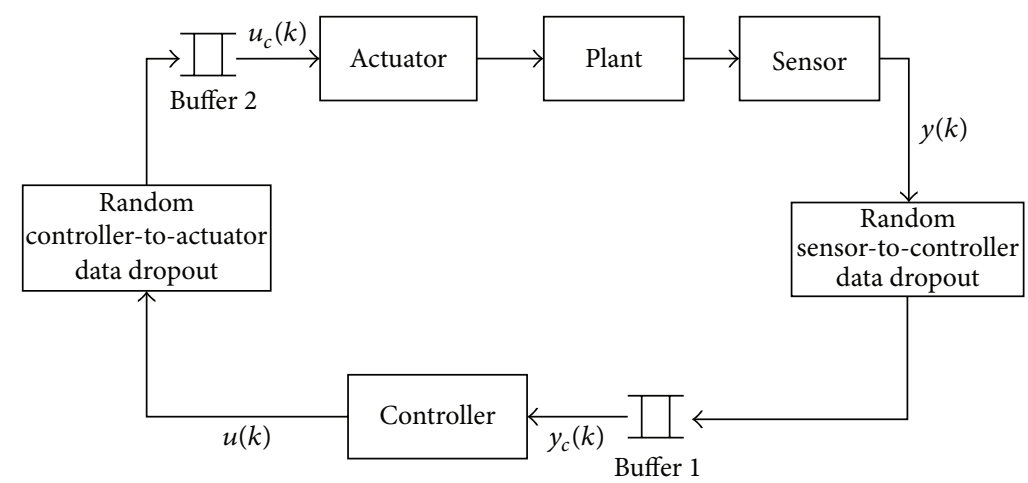

FIGURE 1: Structure of an NCS with random data packet dropouts.

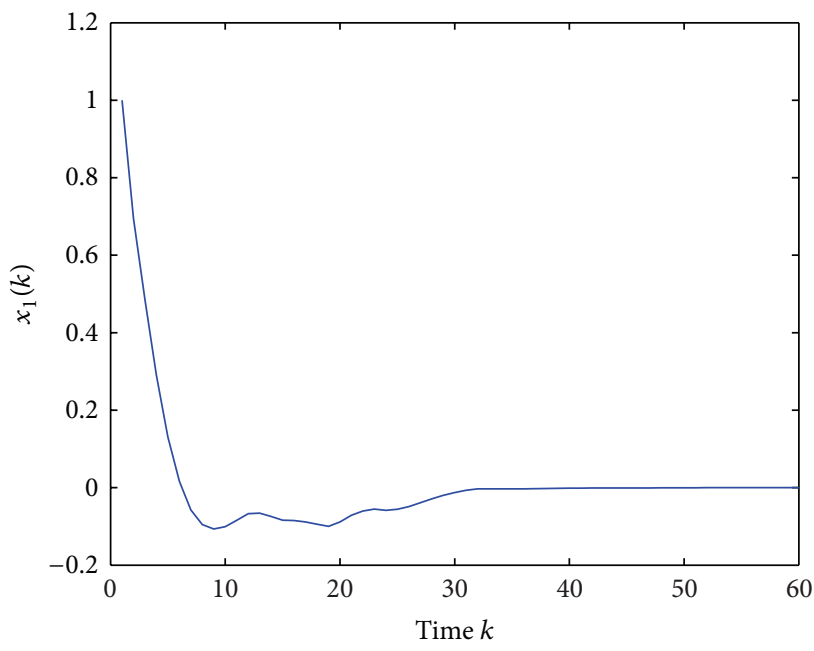

(a)

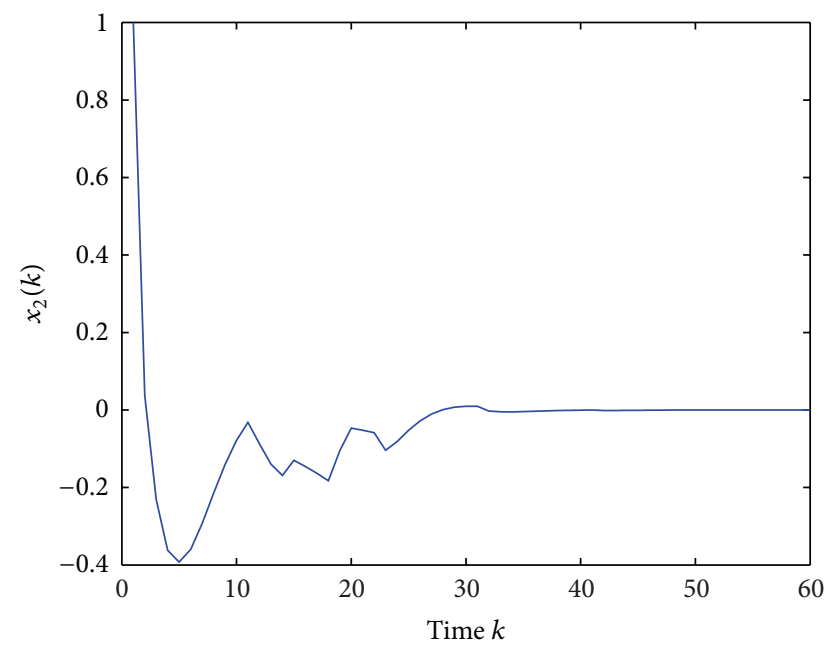

(b)

FIGURE 2: State response with controller in [14]. (a) First component of the state vector $x(k)$. (b) Second component of the state vector $x(k)$.

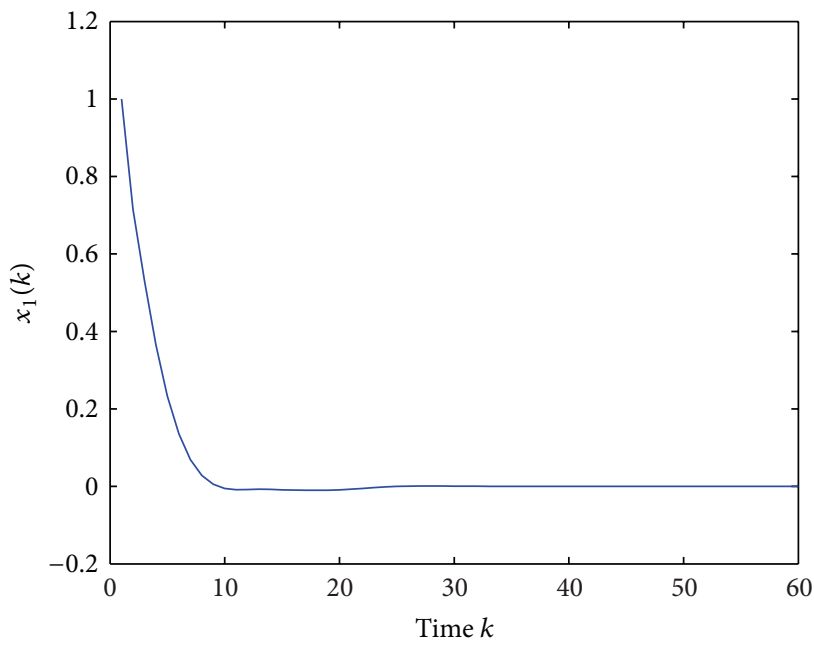

(a)

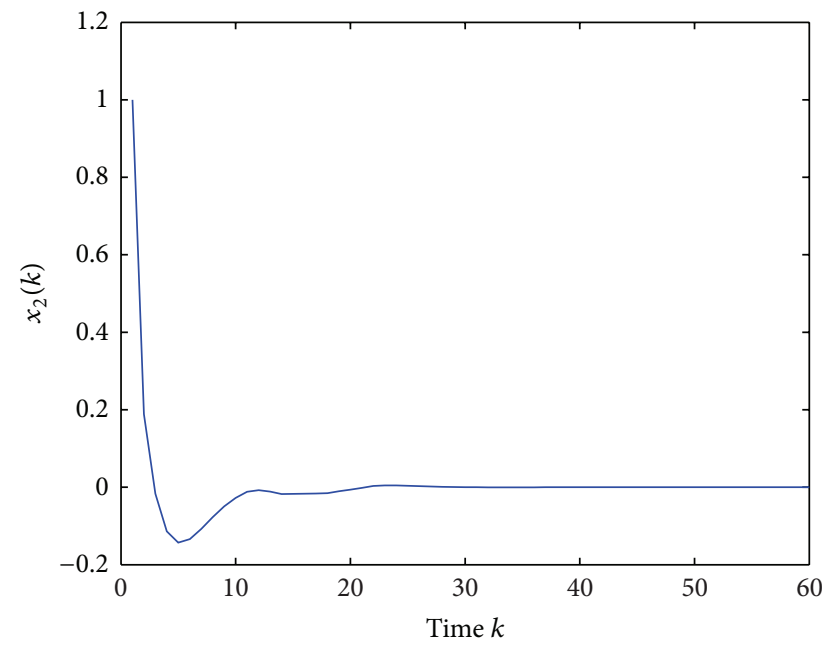

(b)

FIGURE 3: State response with controller proposed in this paper. (a) First component of the state vector $x(k)$. (b) Second component of the state vector $x(k)$. 
where

$$
\begin{gathered}
\Xi_{1}=\left[\begin{array}{cccc}
P A+(1-\bar{\beta}) B G & -(1-\bar{\beta}) B G & 0 & \bar{\beta} P B \\
0 & S A-(1-\bar{\alpha}) H C & 0 & 0 \\
(1-\bar{\alpha}) M C & 0 & \bar{\alpha} M & 0 \\
(1-\bar{\beta}) V \Lambda^{2} V^{T} G & -(1-\bar{\beta}) V \Lambda^{2} V^{T} G & 0 & \bar{\beta} N
\end{array}\right]^{T}, \\
\Xi_{2}=\left[\begin{array}{cccc}
\alpha_{1} B G & -\alpha_{1} B G & 0 & -\alpha_{1} P B \\
\alpha_{2} H C & 0 & -\alpha_{2} H & 0 \\
\alpha_{2} M C & 0 & -\alpha_{2} M & 0 \\
\alpha_{1} V \Lambda^{2} V^{T} G & -\alpha_{1} V \Lambda^{2} V^{T} G & 0 & -\alpha_{1} N
\end{array}\right]^{T},
\end{gathered}
$$

in which $P=U_{1}^{T} P_{1} U_{1}+U_{2}^{T} P_{2} U_{2}, N=V \Lambda P_{1} \Lambda V^{T}$, and $U_{1}, U_{2}, V$, and $\Lambda$ come from (12). Moreover, the controller parameters are given by

$$
\begin{gathered}
K=V \Lambda^{-1} P_{1}^{-1} \Lambda V^{T} G, \\
L=S^{-1} H .
\end{gathered}
$$

Proof. Since there exist $P_{1}>0$, and $P_{2}>0$, such that $P=U_{1}^{T} P_{1} U_{1}+U_{2}^{T} P_{2} U_{2}$, where $U_{1}$, and $U_{2}$ come from (12). It follows from Lemma 3 that there exists a nonsingular matrix $\widetilde{P}=V \Lambda^{-1} P_{1} \Lambda V^{T}$ such that $B \widetilde{P}=P B$. Let $B \widetilde{P}=P B, G=\widetilde{P} K$, and $H=S L$., we can conclude that (28) is equivalent to (14).

\section{Numerical Example}

In this section, an example is used to demonstrate that the controller designed in this paper is effective. We compare our strategy with zero-input strategy [14], which shows that, for systems whose state and input signals change little from one time step to the next, the hold-input strategy adopted in the paper has a better performance.

Consider the following discrete-time unstable system:

$$
\begin{gathered}
A=\left[\begin{array}{cc}
0.7 & 0.2 \\
0 & 1.12
\end{array}\right], \\
B=\left[\begin{array}{c}
-0.03 \\
-1
\end{array}\right], \\
C=\left[\begin{array}{ll}
1 & 0
\end{array}\right] .
\end{gathered}
$$

It is assumed that $\bar{\alpha}=\bar{\beta}=0.1$.

By zero-input strategy in [14], the controller gain and the observer gain are $K=\left[\begin{array}{ll}0.2235 & 0.4312\end{array}\right]$ and $L=$ $\left[\begin{array}{ll}0.9064 & 1.5446\end{array}\right]^{T}$, respectively. Suppose that the initial conditions are $x(0)=\left[\begin{array}{ll}1 & 1\end{array}\right]^{T}, \widehat{x}(0)=\left[\begin{array}{ll}1 & 1\end{array}\right]^{T}$, and $u_{c}(-1)=$ $y_{c}(-1)=0$. We have Figures 2(a) and 2(b). Then, by Theorem 5 of our paper, we obtain $K=\left[\begin{array}{lll}0.1780 & 0.3916\end{array}\right]$ and $L=\left[\begin{array}{ll}0.5770 & 1.1488\end{array}\right]^{T}$. With the same initial conditions, we have Figures 3(a) and 3(b). From Figures 2 and 3 we know that the system can be stabilized by both controllers; however, by comparison, it is easy to know that our controller gives a better performance.
Remark 6. From the system in this example, we can observe that the state, measurement, and input signals change slowly. Moreover, since the latest packet stored in the buffer is used when the $\mathrm{C} / \mathrm{A}$ or $\mathrm{S} / \mathrm{C}$ packet is lost, our controller provides a smoother sequence of inputs. These are the reason why hold-input strategy adopted in the paper gives a better performance.

\section{Conclusion}

In this paper, the problem of observer-based feedback stabilization is considered for NCSs with random packet dropouts. The hold-input strategy is adopted, and sufficient conditions for stochastic stability are derived in the form of linear matrix. Furthermore, the dynamic observer-based control scheme is designed. An example shows that, for systems whose state and input change little from one time step to the next, the holdinput strategy adopted performs better than the zero-input strategy.

\section{Acknowledgments}

This work was supported in part by the National Natural Science Foundation of China under Grant 61202478 and the Fundamental Research Funds for the Central Universities under Grant 2012QNB18.

\section{References}

[1] J. Nilsson, Real-time control systems with delays [Ph.D. thesis], Lund Institute of Technology, Lund, Sweden, 1998.

[2] W. Zhang, M. S. Branicky, and S. M. Phillips, "Stability of networked control systems," IEEE Control Systems Magazine, vol. 21, no. 1, pp. 84-99, 2001.

[3] D. Yue, Q. L. Han, and C. Peng, "State feedback controller design of networked control systems," IEEE Transactions on Circuits and Systems II, vol. 51, no. 11, pp. 640-644, 2004.

[4] H. Wang, X. Liao, and T. Huang, "Accelerated consensus to accurate average in multi-agent networks via state prediction," Nonlinear Dynamics, 2013.

[5] H. Li, X. Liao, and T. Huang, "Second-order locally dynamic consensus of multiagent systems with arbitrarily fast switching directed topologies," IEEE Transactions on Systems, Man, and Cybernetics: Systems, no. 99, pp. 1-11, 2013.

[6] P. V. Zhivoglyadov and R. H. Middleton, "Networked control design for linear systems," Automatica, vol. 39, no. 4, pp. 743750, 2003.

[7] D. Yue, Q.-L. Han, and J. Lam, "Network-based robust $H_{\infty}$ control of systems with uncertainty," Automatica, vol. 41, no. 6, pp. 999-1007, 2005.

[8] L. Zhang, Y. Shi, T. Chen, and B. Huang, "A new method for stabilization of networked control systems with random delays," IEEE Transactions on Automatic Control, vol. 50, no. 8, pp. 11771181, 2005.

[9] H. Gao, T. Chen, and J. Lam, "A new delay system approach to network-based control," Automatica, vol. 44, no. 1, pp. 39-52, 2008.

[10] M. Liu, D. W. C. Ho, and Y. Niu, "Stabilization of Markovian jump linear system over networks with random communication delay," Automatica, vol. 45, no. 2, pp. 416-421, 2009. 
[11] S. P. Wen, Z. Zeng, and T. Huang, "Ho Filtering for neutral systems with mixed delays and multiplicative noises," IEEE Transactions on Circuits and Systems Part II, vol. 59, no. 11, pp. 820-824, 2012.

[12] J. Xiong and J. Lam, "Stabilization of linear systems over networks with bounded packet loss," Automatica, vol. 43, no. 1, pp. 80-87, 2007.

[13] J. Wu and T. Chen, "Design of networked control systems with packet dropouts," IEEE Transactions on Automatic Control, vol. 52, no. 7, pp. 1314-1319, 2007.

[14] Z. Wang, F. Yang, D. W. C. Ho, and X. Liu, "Robust $H_{\infty}$ control for networked systems with random packet losses," IEEE Transactions on Systems, Man, and Cybernetics B, vol. 37, no. 4, pp. 916-924, 2007.

[15] B. Sinopoli, L. Schenato, M. Franceschetti, K. Poolla, M. I. Jordan, and S. S. Sastry, "Kalman filtering with intermittent observations," IEEE Transactions on Automatic Control, vol. 49, no. 9, pp. 1453-1464, 2004.

[16] Z. Wang, F. Yang, D. W. C. Ho, and X. Liu, "Robust finitehorizon filtering for stochastic systems with missing measurements," IEEE Signal Processing Letters, vol. 12, no. 6, pp. 437-440, 2005.

[17] H. Mo and C. N. Hadjicostis, "Feedback control over packet dropping network links," in Proceedings of the Mediterranean Conference on Control and Automation (MED '07), Athens, Greece, July 2007.

[18] S. P. Wen, Z. Zeng, and T. Huang, “Observer-based $H_{\infty}$ control of discrete time-delay systems with random communication packet losses and multiplicative noises," Applied Mathematics and Computation, vol. 219, no. 12, pp. 6484-6493, 2013.

[19] J. G. Li, J. Q. Yuan, and J. G. Lu, "Observer-based $H_{\infty}$ control for networked nonlinear systems with random packet losses," ISA Transactions, vol. 49, no. 1, pp. 39-46, 2010.

[20] L. Schenato, "To zero or to hold control inputs with lossy links?" IEEE Transactions on Automatic Control, vol. 54, no. 5, pp. 1093 1099, 2009.

[21] A. V. Savkin and I. R. Petersen, "Robust filtering with missing data and a deterministic description of noise and uncertainty," International Journal of Systems Science, vol. 28, no. 4, pp. 373378, 1997.

[22] D. W. C. Ho and G. Lu, "Robust stabilization for a class of discrete-time non-linear systems via output feedback: the unified LMI approach," International Journal of Control, vol. 76, no. 2, pp. 105-115, 2003. 


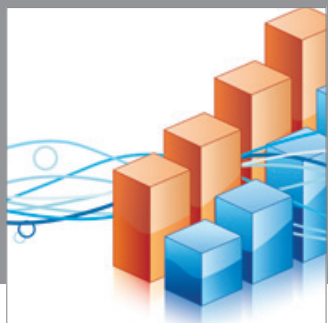

Advances in

Operations Research

mansans

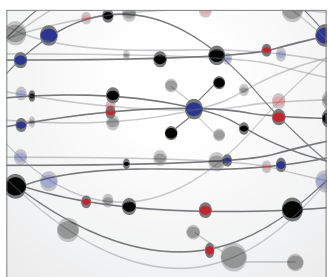

The Scientific World Journal
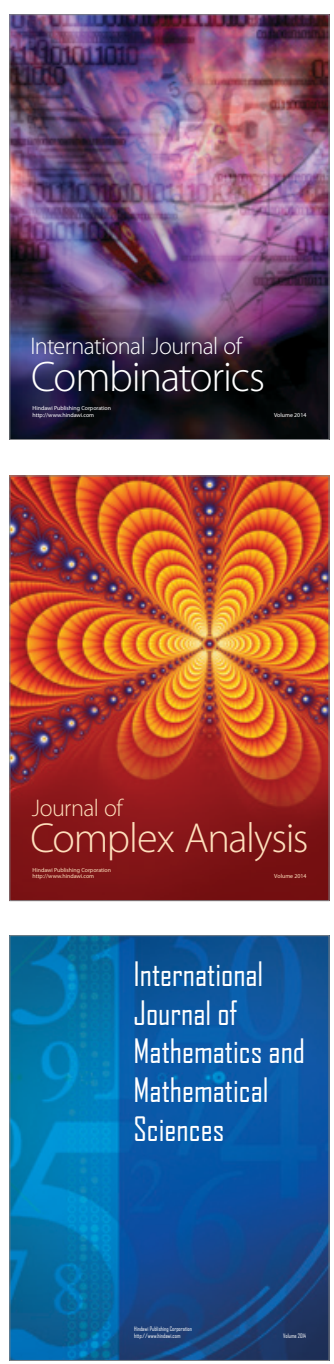
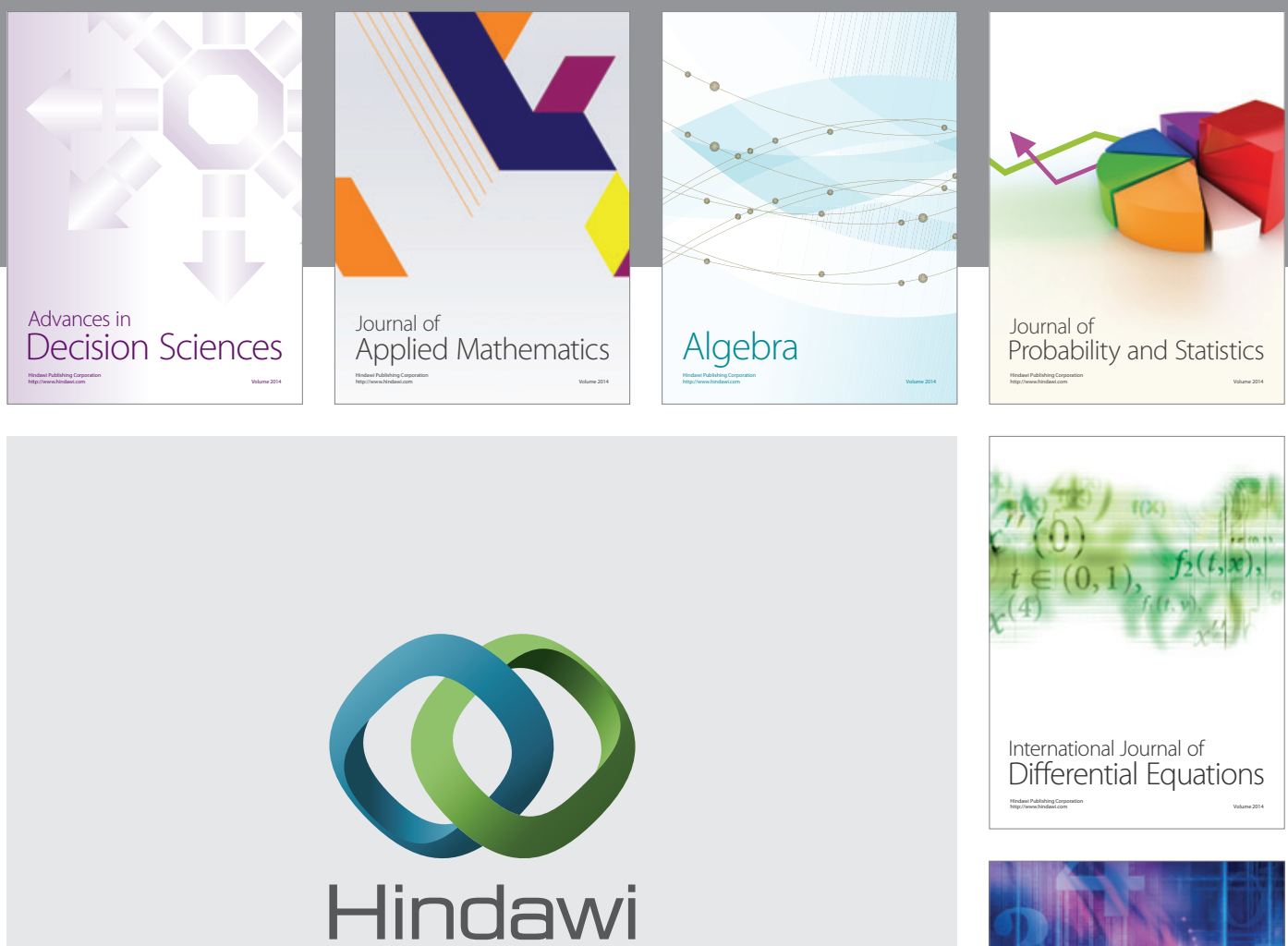

Submit your manuscripts at http://www.hindawi.com
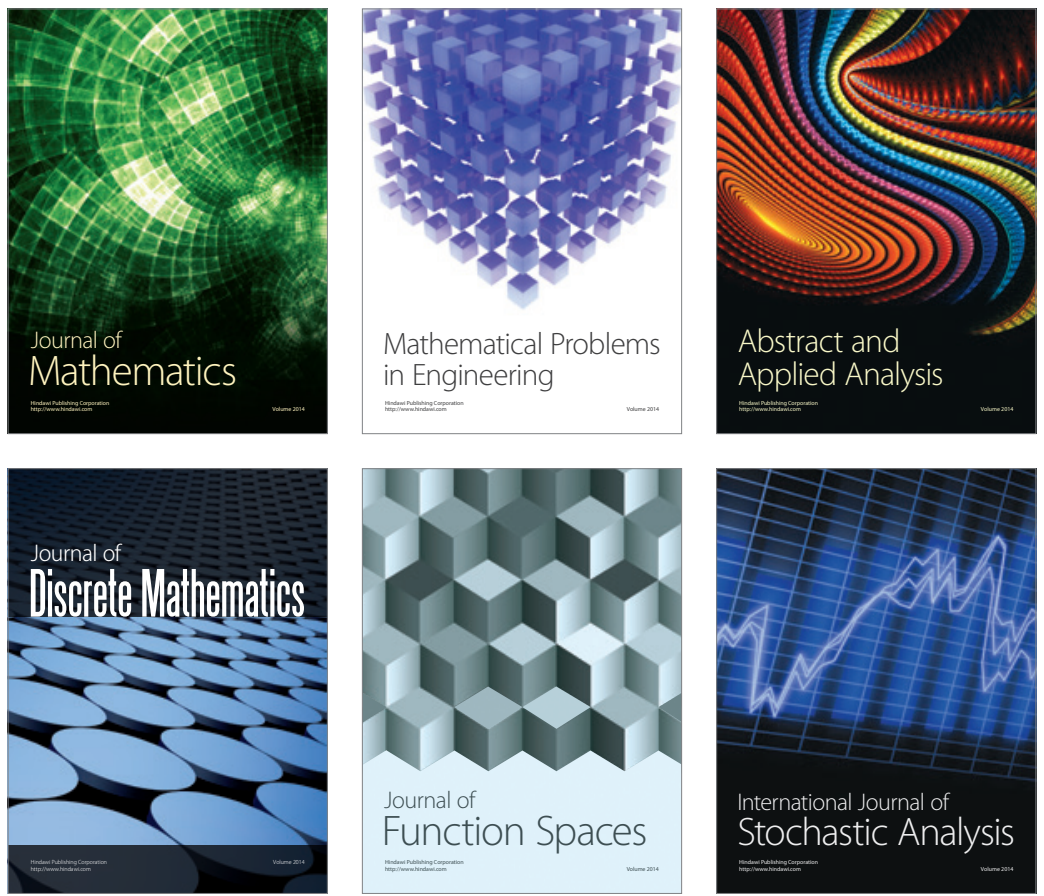

Journal of

Function Spaces

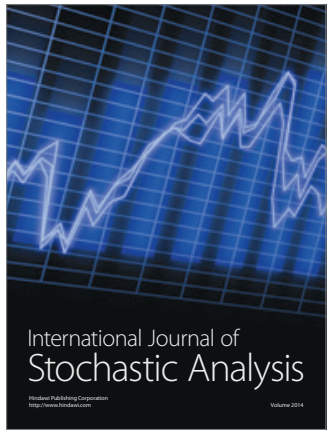

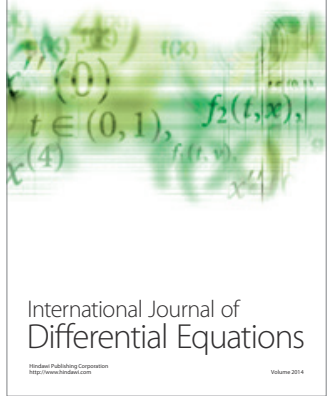
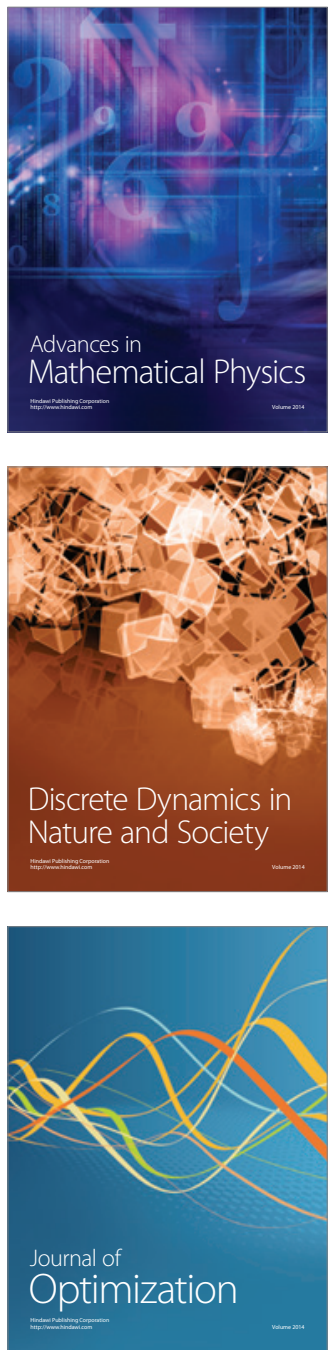